\title{
CONVERGENCE OF NONCONFORMING FINITE ELEMENT APPROXIMATIONS TO FIRST-ORDER LINEAR HYPERBOLIC EQUATIONS
}

\author{
NOEL J. WALKINGTON
}

\begin{abstract}
Finite element approximations of the first-order hyperbolic equation $\mathbf{U} \cdot \nabla u+\alpha u=f$ are considered on curved domains $\Omega \subset \mathbb{R}^{2}$. When part of the boundary of $\Omega$ is characteristic, the boundary of numerical domain, $\Omega_{h}$, may become either an inflow or outflow boundary, so it is necessary to select an algorithm that will accommodate this ambiguity.

This problem was motivated by a problem in acoustics, where an equation similar to the one above is coupled to three elliptic equations. In the last section, the acoustics problem is briefly recalled and our results for the first-order equation are used to demonstrate convergence of finite element approximations of the acoustics problem.
\end{abstract}

\section{INTRODUCTION}

In this paper we address some technical issues associated with approximating a first-order spatially hyperbolic equation. This problem was motivated by a problem in linear acoustics which gave rise to a system of coupled equations, one of whose principal parts was first-order in space. The acoustics problem was naturally posed in a domain, $\Omega \subset \mathbb{R}^{2}$, where a fluid entered one part of the boundary (the inflow) and exited through another portion of the boundary (the outflow). The remainder of the boundary was tangential to the mean flow, and the streamlines of this mean flow were the characteristics of a first-order equation of the form

$$
\mathbf{U} \cdot \nabla u+\alpha u=f,
$$

where $f$ represents the coupling terms and $\mathbf{U}$ is a known (mean flow) velocity field.

The numerical approximation of the solution of such problems requires triangulation of the domain $\Omega$, the union of the triangles giving a domain $\Omega_{h}$ which approximates $\Omega$. One problem encountered with this approach is that portions of the boundary of $\Omega_{h}$ may no longer be characteristic where the corresponding portions of $\Omega$ are. This may result in triangles having one side which contains both inflow and outflow regions of the mean flow, and if the

Received November 26, 1990; revised February 12, 1991.

1991 Mathematics Subject Classification. Primary 76Q05, 65N30.

Key words and phrases. Hyperbolic equations, nonconforming approximation.

The author was supported by the National Science Foundation Grant No. DMS-9002768 while this work was undertaken. 


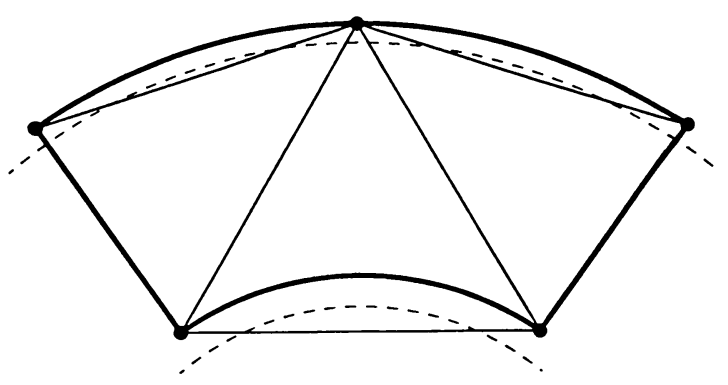

FIGURE 1

Triangulation of a curved domain

first-order equation is to be well posed on such a domain, boundary conditions must be applied on each inflow boundary. A gross example of this is shown in Figure 1, where a duct with curved sides is triangulated with three elements. The two dotted lines represent streamlines of the mean flow. Clearly the base of the middle triangle becomes both an inflow and outflow boundary (assuming the mean flow extends smoothly outside $\Omega$ ), as do the tops of the left and right triangles.

In order to circumvent the problem with inflow and outflow boundaries, we will approximate the given mean flow with one that has streamlines parallel to the appropriate portions of the numerical boundary $\Omega_{h}$. The approximation we use arises naturally from the finite element procedure used to construct $\Omega_{h}$. In order to compare the approximate solution on $\Omega_{h}$ with the exact solution on $\Omega$, we show that there is a map from $\Omega_{h}$ to $\Omega$, and when the approximate solutions are composed with this map, they converge to the exact solution.

Various algorithms have been proposed for the solution of first-order hyperbolic equations; however, it has always been assumed that the domain was exactly triangulated. Also, it is sometimes assumed that the side of any triangle is never characteristic. These assumptions are incompatible for certain fluid flow problems, since the domain is bounded by streamlines which are the characteristics of the vorticity transport equation. It is known that simplistic Galerkin approximations of hyperbolic equations do not produce good algorithms, so most algorithms use some form of modified Galerkin approximation. The discontinuous Galerkin method $[8,7]$ is one such successful modification. This algorithm approximates the solution using discontinuous test and trial functions. A continuous variant of this technique has been developed [4] where the trial functions are continuous, but the test functions are discontinuous and constructed from lower-degree polynomials. Another approach, motivated from upwind difference techniques, is to perturb the standard Galerkin method by 'adjusting' the test functions. Typically, the test space is modified so that it contains some of the derivatives of the trial functions. For example, Winther [12] constructs a scheme where the derivatives of the trial space form the test space. Similarly, streamwise diffusion algorithms $[6,10]$ include various derivatives in the test functions, which essentially correspond to an artificial viscosity in the streamwise direction. The modified method of characteristics introduced in [3] uses a Galerkin procedure for the spatial variables and uses characteristic directions to construct difference quotients for the temporal discretization. 
When constructing algorithms for the solution of hyperbolic problems, it is highly desirable to take advantage of the hyperbolic nature, constructing the solution step by step with a marching process that eventually exhausts the whole domain. However, if the hyperbolic equation is coupled to an elliptic equation (or is part of a convection-diffusion equation), this process will not work, and all quantities will have to be solved for simultaneously. While the algorithm introduced in $\S 2$ can be used as a marching procedure, it was motivated by the desire to make the bookkeeping associated with the hyperbolic equation identical to that of the elliptic equations to which it is coupled (i.e., standard finite element assembly techniques). The algorithm considered below could be thought of either as a limiting case of a streamwise diffusion algorithm, or as approximating the second-order equation obtained by taking the streamwise derivative of the given first-order hyperbolic equation. The solution of a firstorder hyperbolic equation may be discontinuous across the characteristics, and since the streamlines of the mean flow (i.e., characteristics) are known a priori, finite elements are aligned with them. This enables the construction of trial functions which admit discontinuities across a finite number of streamlines. Since practical acoustics problems typically involve rough data, it is expected that discontinuous approximations will perform better on coarse meshes than their continuous counterparts.

In the following, $\Omega \subset \mathbb{R}^{2}$ is a simply connected Lipschitz domain. $W^{m, p}(\Omega)$ denotes the Sobolev space of functions with $m$ derivatives $p$-integrable, with the Sobolev norm denoted $\|\cdot\|_{m, p, \Omega}$, and the seminorm denoted $|\cdot|_{m, p, \Omega}$. $H^{m}(\Omega)$ is the Hilbert space $W^{m, 2}(\Omega) . \mathscr{P}_{k}(\widehat{T})$ is the linear space of polynomials of degree less than or equal to $k$ defined on $\widehat{T}$, and $C^{k}(\Omega)$ is the Banach space of functions with $k$ continuous bounded derivatives on $\Omega$. Constants will be denoted generically by $C$ or $c$, typically $C$ denoting an upper bound, and $c$ denoting a lower bound. All constants will be independent of the mesh parameter $h$ which corresponds to the maximum diameter of the triangles used to triangulate $\Omega .|\cdot|$ will be used to denote the Euclidean norm of vectors in $\mathbb{R}^{2}$ and the norm of a linear transformation (matrix) from $\mathbb{R}^{2}$ to itself, and a dot, $\cdot$, denotes the inner product on $\mathbb{R}^{2}$.

In the next section we isolate the hyperbolic equation that arose in the acoustics problem by ignoring the coupling terms, and consider numerical approximation of the solution. In $\S 3$ the full acoustics problem is recalled, and the techniques of $\S 2$ are utilized to study the convergence of numerical solutions.

\section{MODEL PROBLEM}

We consider the following model problem similar to the hyperbolic equation that arose in a plane acoustics problem [11]:

$$
\mathbf{U} \cdot \nabla u+\alpha u=f \quad \text { in } \Omega,\left.\quad u\right|_{\Gamma_{1}}=0,
$$

where $\Omega \subset \mathbb{R}^{2}$ is a bounded simply connected Lipschitz domain with boundary $\partial \Omega=\Gamma_{1} \cup \Gamma \cup \Gamma_{2}$. It will be assumed that the components of $\Gamma$ are parallel to the streamlines of $\mathbf{U}$, while $\Gamma_{1}$ and $\Gamma_{2}$ are inflow and outflow boundaries of $\mathbf{U}$ respectively (see Figure 2). Equation (1) describes convection of a quantity $u$ by the velocity field $\mathbf{U}$, and is ubiquitous in mathematical modeling of transport phenomena (in [11], $u$ corresponds to vorticity, and is transported along 


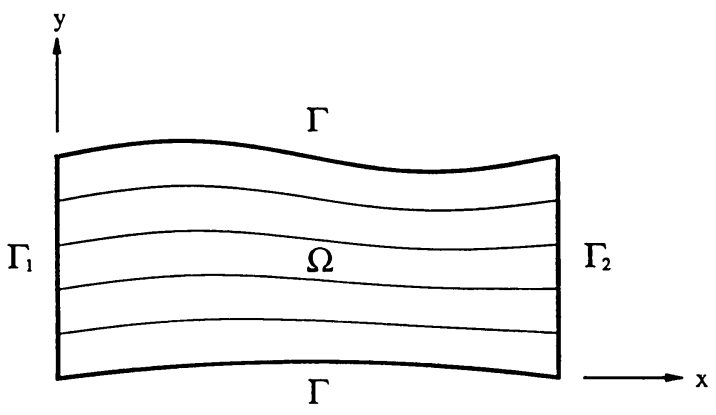

FIGURE 2

Duct and streamlines

streamlines of the flow by a classical theorem of Helmholtz). The streamlines of the flow $\mathbf{U}$ are the characteristics of (1), so if $\Omega$ is partitioned into subdomains by a collection of streamlines, the solution can be constructed as the amalgamation of the solutions on each subdomain. Since the numerical scheme to be described below preserves this property, we assume that $\Gamma_{1}$ consists of exactly one component.

It is natural to introduce the streamwise coordinate system, $(x(s, t), y(s, t))$ $=\mathbf{X}(s, t)$ defined by $\partial \mathbf{X} / \partial s=\mathbf{U}, \mathbf{X}(0, t)=\mathbf{x}(t)$ on $\Gamma_{1}$, where $\Gamma_{1}$ is parameterized by its arc length $t$. Clearly, equation (1) reduces to

$$
\frac{\partial u}{\partial s}+\alpha u=f \quad \text { in } \widehat{\Omega}=\mathbf{X}^{-1}(\Omega) .
$$

In order to have a well-defined coordinate system, we will henceforth assume that $\mathbf{U}$ is Lipschitz and $|\mathbf{U}| \geq c_{0}>0$ throughout $\bar{\Omega}$. An elementary argument outlined in the Appendix shows that $\mathbf{X}$ is a homeomorphism. In the sequel, it will be assumed that $\mathbf{X}$ is a $C^{k}$ diffeomorphism with Jacobian bounded away from zero whenever $\mathbf{U} \in C^{k}(\Omega)$. In the Appendix, it is shown that if the angle between the mean flow $U$ and the tangent to $\Gamma_{1}$ is bounded away from zero, a.e., then this assumption holds. ${ }^{1}$ It is also shown that the nontangential restriction can be relaxed, allowing $U$ to be tangential to $\Gamma_{1}$ at isolated points.

It is natural to seek a solution of equation (1), $u$, whose streamwise derivative $\mathrm{U} \cdot \nabla u$ is in $L^{2}(\Omega)$. Accordingly, we define the Hilbert space $U$ to be the completion of the set $\left\{u \in C^{1}(\bar{\Omega})|u|_{\Gamma_{1}}=0\right\}$ in the norm $\|u\|_{L^{2}(\Omega)}+\|\mathbf{U} \cdot \nabla u\|_{L^{2}(\Omega)}$. A Poincare inequality of the form $\|u\|_{L^{2}(\widehat{\Omega})} \leq C\|\partial u / \partial s\|_{L^{2}(\widehat{\Omega})}$ trivially holds in $\widehat{\Omega}=\mathbf{X}^{-1}(\Omega)$. Pulling this estimate back through $\mathbf{X}$ shows that $\|u\|_{L^{2}(\Omega)} \leq$ $C\|\mathbf{U} \cdot \nabla u\|_{L^{2}(\Omega)}$, so we may take the norm on $U$ to be $\|u\|_{U}=\|\mathbf{U} \cdot \nabla u\|_{L^{2}(\Omega)}$. The following weak form of equation (1) was introduced in [11]:

$$
u \in U, \quad \int_{\Omega}\{\mathbf{U} \cdot \nabla u+\alpha u\}(\mathbf{U} \cdot \nabla v)=\int_{\Omega} f(\mathbf{U} \cdot \nabla v) \quad \forall v \in U .
$$

An elementary calculation shows that $C_{0}^{\infty}(\Omega) \subset\{\mathbf{U} \cdot \nabla u \mid u \in U\}$, so that solutions of (3) are distributional solutions of (1) when written as

$$
\nabla \cdot(\mathbf{U} u)-(\nabla \cdot \mathbf{U}) u+\alpha u=f .
$$

\footnotetext{
${ }^{1}$ In this situation, the initial condition for $\mathbf{X}(0, t)$ is specified on a smooth approximation to $\Gamma_{1}$, so that $\Gamma_{1}$ may no longer correspond to $s=0$.
} 
In the Appendix, equation (1) is solved by integrating along the characteristics. However, when coupled to elliptic equations, this procedure is not suitable from a numerical point of view. It is for this reason that we consider the above weak problem. In [11], it was necessary to assume that $\alpha$ was small, and in this situation the traditional Lax-Milgram theorem shows (3) is well posed. The next theorem shows that this restriction is not necessary in general.

Theorem 1. Suppose that the map $\mathbf{X}$ constructed above is a $C^{1}$ diffeomorphism with Jacobian bounded away from zero. Let the coefficient $\alpha \in L^{\infty}(\Omega)$, and $f \in U^{\prime}$; then Problem (3) is well posed.

Proof. Define the bilinear form on $U$ by

$$
a(u, v)=\int_{\Omega}\{\mathbf{U} \cdot \nabla u+\alpha u\}(\mathbf{U} \cdot \nabla v) .
$$

The form $a$ is clearly continuous, so the theorem will follow provided $a$ is coercive, i.e.,

$$
\sup _{v \neq 0} \frac{a(u, v)}{\|v\|_{U}} \geq c\|u\|_{U}, \quad \sup _{u} a(u, v)>0 \text { for } v \neq 0 .
$$

Fix $u \in U$; since $L^{2}(\Omega) \subset\{\mathbf{U} \cdot \nabla v \mid v \in U\}$, we may choose $\mathbf{U} \cdot \nabla v=f=$ $\mathrm{U} \cdot \nabla u+\alpha u$ to get $a(u, v)=\|f\|_{L^{2}(\Omega)}^{2}=\|f\|_{L^{2}(\Omega)}\|v\|_{U}$. The existence result for equation (1) given in the Appendix shows that $\|u\|_{U} \leq C\|f\|_{L^{2}(\Omega)}$, so that $a(u, v) \geq(1 / C)\|u\|_{U}\|v\|_{U}$, and the first coercivity condition holds.

For $v \in U, v \neq 0$ fixed, select $u \in U$ as the solution to $\mathbf{U} \cdot \nabla u+\alpha u=\mathbf{U} \cdot \nabla v$ to show that $a(u, v)=\|v\|_{U}^{2}>0$.

\subsection{Regularity. Integration of equation (2) gives}

$$
u(s, t)=u(0, t)+\int_{0}^{s}\{\hat{f}(\xi, t)-\hat{\alpha}(\xi, t) u(\xi, t)\} d \xi \quad \text { in } \widehat{\Omega}=\mathbf{X}^{-1}(\Omega),
$$

where $\hat{f}=f \circ \mathbf{X}$ and $\hat{\alpha}=\alpha \circ \mathbf{X}$. Gronwall's inequality may be used to justify formal calculations of the form

$$
\frac{\partial u}{\partial t}(s, t)=\frac{\partial u}{\partial t}(0, t)+\int_{0}^{s}\left\{\frac{\partial \hat{f}}{\partial t}(\xi, t)+\frac{\partial \hat{\alpha}}{\partial t}(\xi, t) u(\xi, t)+\hat{\alpha}(\xi, t) \frac{\partial u}{\partial t}(\xi, t)\right\} d \xi
$$

which shows that both $u$ and $\partial u / \partial s$ are in $H^{k}(\widehat{\Omega})$ provided $\hat{f} \in H^{k}(\widehat{\Omega})$ and $\hat{\alpha} \in W^{k, \infty}(\widehat{\Omega})$. This will imply that both $u$ and $\mathbf{U} \cdot \nabla u$ are in $H^{k}(\Omega)$ provided $f \in H^{k}(\Omega), \alpha \in W^{k, \infty}(\Omega), u(0, t) \in H^{k}\left(\Gamma_{1}\right)$ and $\mathbf{U} \in C^{k}(\Omega)$ with $|\mathbf{U}| \geq c_{0}>0$.

2.2. Finite element approximations. Since the mean flow $\mathbf{U}$ is known a priori, it is possible to generate the streamlines and position the finite element nodes on them. If the element boundaries were perfectly aligned with the streamlines, it would be possible to let the basis functions be discontinuous across them. In practice, the element boundaries will be polynomial approximations to the streamlines, so discontinuous basis functions will give rise to a nonconforming approximation. A second complication is the requirement that a Poincare inequality hold for the discrete space with constant independent of the mesh size $h$. This is achieved by approximating the velocity field $\mathbf{U}$ by one whose streamlines are parallel to the element boundaries. 


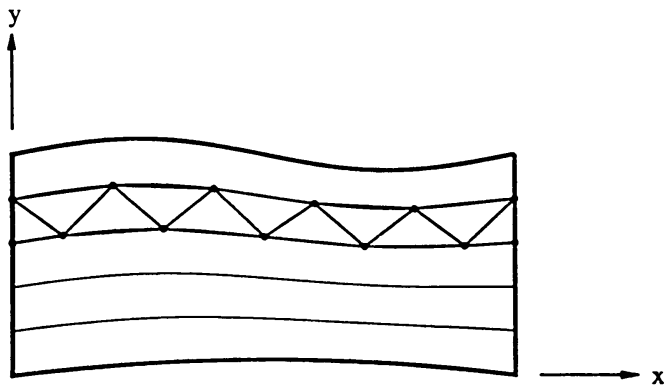

FIGURE 3a

Approximating a streamtube
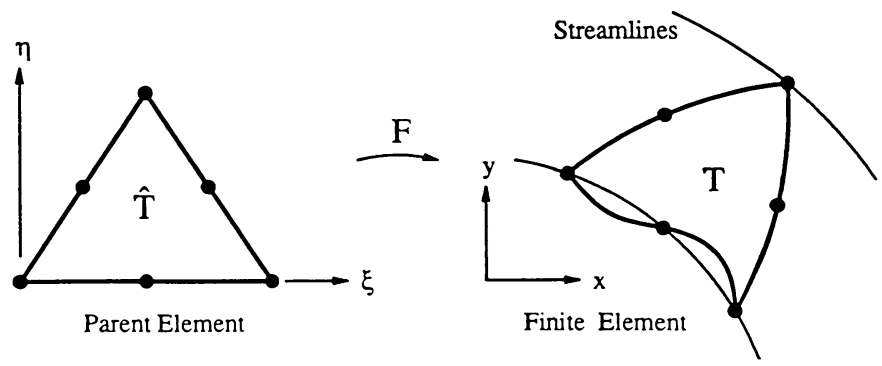

FIGURE 3b

Aligning elements with streamlines

In the sequel, we will limit the discussion to linear or quadratic isoparametric elements $(k=1$ or $k=2$ below). The arguments presented will apply equally well to either triangular or quadrilateral elements; however, we will use the terminology appropriate to triangular elements. We adopt the following notation, taken from Ciarlet [2, Chapters 3-4]: $\Omega_{h}$ is the approximation to $\Omega$ given by the union of all the finite elements; a generic isoparametric mapping from the parent element $\widehat{T}$ to a typical finite element $T$ is denoted by $F: \widehat{T} \rightarrow T$; if $u$ is defined on a finite element $T$, then $\hat{u}$ is defined on the parent element $\widehat{T}$ by $\hat{u}(\xi, \eta)=u \circ F(\xi, \eta) ; F_{\xi}=\partial F / \partial \xi$ denotes the partial derivative of $F$ with respect to $\xi$ etc.

We will consider finite element meshes, $\mathscr{T}_{h}$, constructed so that one boundary of each element approximates a streamline, with elements aligned along stream tubes as indicated in Figure 3. Moreover, we will choose the element mappings so that the side of the parent element lying on the $\xi$-axis maps to the side of the finite element on the streamline, as shown in Figure 3. Following [2, p. 124], we define a finite family of meshes to be regular if the ratio of the element diameter to the diameter of the largest inscribed sphere for each element is bounded, and, for quadratic elements, the midside nodes are located within a distance $C h^{2}$ from the midside locations predicted by the affine map determined by the corner nodes. The following lemma is taken from [2, p. 237].

Lemma 2. Let $\left\{\mathscr{T}_{h}\right\}_{h>0}$ be a regular family of meshes; then there are constants $c$ and $C$, independent of $h$, such that

1. $c h^{2} \leq J_{F} \leq C h^{2} \quad\left(J_{F}\right.$ is the Jacobian of $\left.D F\right)$,

2. $c h \leq\left|F_{\xi}\right|,\left|F_{\eta}\right|$, and $|D F| \leq C h$,

3. $\left|D^{2} F\right| \leq C h^{2}$. 
We approximate $\mathbf{U}$ on each element by $\overline{\mathbf{U}}=\left(|\mathbf{U}| /\left|F_{\xi}\right|\right) F_{\xi}$ and introduce the space

$$
\begin{aligned}
U_{h}=\left\{u: \Omega_{h} \rightarrow \mathbb{R}|u|_{T}\right. & =\hat{u} \circ F^{-1}, \hat{u} \in \mathscr{P}_{k}(\widehat{T}), \forall T \in \mathscr{T}_{h}, \\
\left.u\right|_{\Gamma_{1}} & =0, u \text { continuous in each approximation stream tube }\} .
\end{aligned}
$$

Note that this construction yields $\overline{\mathbf{U}}$ tangential to the approximate stream tube boundaries. Weak problem (3) is then approximated as

$$
u_{h} \in U_{h}, \quad \int_{\Omega_{h}}\left\{\overline{\mathbf{U}} \cdot \nabla u_{h}+\alpha u_{h}\right\}\left(\overline{\mathbf{U}} \cdot \nabla v_{h}\right)=\int_{\Omega_{h}} f\left(\overline{\mathbf{U}} \cdot \nabla v_{h}\right) \quad \forall v_{h} \in U_{h} .
$$

Of course, it is assumed that $\alpha$ and $f$ are extended suitably to $\Omega_{h}$ (see Theorem (12)). The nonstandard feature of this problem is that even when $\Omega_{h}=\Omega$, $\|\cdot\|_{U}$ and $\|\cdot\|_{U_{h}}$ do not agree on $U \cap U_{h}$.

The solution of (4) can be accomplished in a step-by-step fashion along the approximate streamtubes, beginning with the triangle at the inflow boundary. To see this, it suffices to show that given a test function $v_{h} \in U_{h}$, it is possible to construct another test function $w_{h}$ such that for any triangle $T,\left.\overline{\mathrm{U}} \cdot \nabla v_{h}\right|_{T}=$ $\left.\overline{\mathbf{U}} \cdot \nabla w_{h}\right|_{T}$, and $\overline{\mathbf{U}} \cdot \nabla w_{h}=0$ elsewhere. In this situation the problem decouples into subproblems on each triangle. In order to demonstrate this property of the test functions, consider the parent element $\widehat{T}$ of the element $T$ in question. Given the values of $v_{h}$ on the left hand (inflow) boundary, they can be extended to the whole triangle so that they are constant with respect to $\xi$. This function will be a polynomial of degree $\leq k$ when $v_{h} \in U_{h}$. Subtracting this function from $v_{h}$ gives another test function $w_{h}$ which vanishes on the inflow boundary and satisfies $\partial w_{h} / \partial \xi=\partial v_{h} / \partial \xi$ on $\hat{T}$. The function $w_{h}$ can be extended to zero on all triangles in the streamtube preceding $T$, and can be extended so that $\partial w_{h} / \partial \xi=0$ on all triangles after $T$. Since $\overline{\mathbf{U}} \cdot \nabla v_{h}=\left(|\mathbf{U}| /\left|F_{\xi}\right|\right) \partial v_{h} / \partial \xi$, the result follows.

In order to limit the amount of technical detail, we will assume that all integrals are computed exactly, and that $\Gamma_{1}$ and $\Gamma_{2}$ are exactly interpolated by the finite element mesh. The techniques in Ciarlet [2, Chapter 4] are applicable if either of these assumptions is violated. The next lemma shows that, like the space $U$, the space $U_{h}$ enjoys a Poincaré inequality, with constant independent of $h$.

Lemma 3. Let $\left\{\mathscr{T}_{h}\right\}_{h>0}$ be a regular family of meshes aligned with the streamlines of $\mathbf{U}$ as described above. If $\mathbf{U} \geq c_{0}>0$ throughout $\boldsymbol{\Omega}$, then there is a constant $C$ independent of mesh such that $\|u\|_{L^{2}\left(\Omega_{h}\right)} \leq C\|\overline{\mathbf{U}} \cdot \nabla u\|_{L^{2}\left(\Omega_{h}\right)}$.

Proof. We begin by considering the $L^{2}$ norm of $u$ on a finite element $T$. Let $\xi_{1}(\eta)$ and $\xi_{2}(\eta)$ be the linear functions describing the two sides of the parent element $\widehat{T}$ that do not lie on the $\xi$-axis (see Figure 3 ); then

$$
u^{2}(\xi, \eta)=u^{2}\left(\xi_{1}(\eta), \eta\right)+\int_{\xi_{1}(\eta)}^{\xi} \frac{\partial}{\partial \xi} u^{2}(\chi, \eta) d \chi .
$$

Recalling that the Jacobian satisfies $c h^{2} \leq J_{F}(\xi, \eta) \leq C h^{2}$, we may multiply the above identity by $C h^{2}$ to obtain

$$
\|u\|_{L^{2}(T)}^{2} \leq C\left[h^{2}\|u\|_{L^{2}\left(C_{1}\right)}^{2}+\frac{2}{c}\|u\|_{L^{2}(T)}\left\|\frac{\partial u}{\partial \xi}\right\|_{L^{2}(T)}\right],
$$


where

$$
\|u\|_{L^{2}\left(C_{1}\right)}^{2}=\int_{0}^{1} u^{2}\left(\xi_{1}(\eta), \eta\right) d \eta,
$$

and we have assumed that $\widehat{T}$ has a base of unit length and is of unit height. A similar calculation show that

$$
h^{2}\left(\|u\|_{L^{2}\left(C_{2}\right)}^{2}-\|u\|_{L^{2}\left(C_{1}\right)}^{2}\right) \leq \frac{2}{c}\|u\|_{L^{2}(T)}\left\|\frac{\partial u}{\partial \xi}\right\|_{L^{2}(T)} .
$$

If we let $G$ denote a typical streamtube, summing over all the elements in $G$, and recalling that $\left.u\right|_{\Gamma_{1}}=0$, gives

$$
h^{2}\|u\|_{L^{2}\left(C_{i}\right)}^{2} \leq \frac{2}{c} \sum_{T \subset G}\|u\|_{L^{2}(T)}\left\|\frac{\partial u}{\partial \xi}\right\|_{L^{2}(T)},
$$

where $C_{i}$ is any of the interelement boundaries within a streamtube. Letting $N_{G}$ denote the number of elements with the streamtube $G$, we obtain

$$
\|u\|_{L^{2}(G)}^{2}=\sum_{T \subset G}\|u\|_{L^{2}(T)}^{2} \leq \frac{2 C}{c}\left(N_{G}+1\right) \sum_{T \subset G}\|u\|_{L^{2}(T)}\left\|\frac{\partial u}{\partial \xi}\right\|_{L^{2}(T)} .
$$

The definition of $\overline{\mathbf{U}}$ reveals $\partial u / \partial \xi=\left(\left|F_{\xi}\right| /|\mathbf{U}|\right) \overline{\mathbf{U}} \cdot \nabla u$, so that

$$
\|u\|_{L^{2}(G)} \leq \frac{2 C}{c_{0} c}\left(N_{G}+1\right) \max _{T \subset G}\left(\left\|F_{\xi}\right\|_{L^{\infty}(T)}\right)\|\overline{\mathbf{U}} \cdot \nabla u\|_{L^{2}(G)} .
$$

The theorem now follows from the estimates $N_{G} \leq C / h$ and $\left|F_{\xi}\right| \leq C h$.

Equation (5) in the above proof reveals the following local Poincare estimate.

Corollary 4. Suppose $u \in U_{h}$ vanishes on the inflow boundary of a triangle; then

$$
\|u\|_{L^{2}(T)} \leq C h\|\overline{\mathbf{U}} \cdot \nabla u\|_{L^{2}(T)},
$$

where $C$ is independent of $h$ and $T$.

In order to show that the approximate problem has a solution, it suffices to show that it can be constructed in a step-by-step fashion. However, in order to establish error estimates, it is convenient to use the Lax-Milgram theorem.

Theorem 5. Let $\left\{\mathscr{T}_{h}\right\}_{h>0}$ be a regular family of meshes aligned with the streamlines of $\mathbf{U} \in C^{1}(\boldsymbol{\Omega})$ as described above. If $\mathbf{U} \geq c_{0}>0$ throughout $\Omega$, $\alpha \in L^{\infty}(\Omega)$, then there exists $h_{0}>0$ such that if $h<h_{0}$, the approximate weak problem (4) satisfies the hypotheses of the Generalized Lax-Milgram Theorem with coercivity constant independent of $h$.

Proof. As with the continuous problem, we consider the bilinear form $a_{h}$ : $U_{h} \times U_{h} \rightarrow \mathbb{R}$ given by

$$
a_{h}(u, v)=\int_{\Omega}\{\overline{\mathbf{U}} \cdot \nabla u+\alpha u\}(\overline{\mathbf{U}} \cdot \nabla v) .
$$

In order to establish the first coercivity condition, we consider $u \in U_{h}$ fixed, and use the Riesz Theorem to select $v \in U_{h}$ as the solution to

$$
\int_{\Omega}(\overline{\mathbf{U}} \cdot \nabla v)(\overline{\mathbf{U}} \cdot \nabla w)=\int_{\Omega}\{\overline{\mathbf{U}} \cdot \nabla u+\alpha u\}(\overline{\mathbf{U}} \cdot \nabla w) \quad \forall w \in U_{h} .
$$


Putting $w=v$ in the above shows that $a_{h}(u, v)=\|v\|_{U_{h}}^{2}$. It remains to show that there is a constant $C>0$ independent of $h$ such that $\|u\|_{U_{h}} \leq C\|v\|_{U_{h}}$, which implies $a(u, v) \geq(1 / C)\|u\|_{U_{h}}\|v\|_{U_{h}}$. Putting $w=u$ into the above expression for $v$ yields

$$
\|u\|_{U_{h}} \leq\|v\|_{U_{h}}+\|\alpha\|_{L^{\infty}(\Omega)}\|u\|_{L^{2}\left(\Omega_{h}\right)},
$$

so that it suffices to show that

$$
\|u\|_{L^{2}\left(\Omega_{h}\right)} \leq C\left(\|v\|_{U_{h}}+h\|u\|_{U_{h}}\right)
$$

To establish this inequality, we use a discrete Gronwall type inequality, whose construction is rather detailed.

We begin by recalling that equation (6) decouples into separate equations over each element. Letting $\widehat{T}$ be the parent element, each equation takes the form

$$
\int_{\widehat{T}}\left(\frac{|\mathbf{U}|}{\left|F_{\xi}\right|} \frac{\partial u}{\partial \xi}+\alpha u\right) \frac{|\mathbf{U}|}{\left|F_{\xi}\right|} w J=\int_{\widehat{T}} \frac{|\mathbf{U}|^{2}}{\left|F_{\xi}\right|^{2}} \frac{\partial v}{\partial \xi} w J \quad \forall w \in \mathscr{P}_{k, \xi}(\widehat{T}),
$$

where $\mathscr{P}_{k, \xi}(\widehat{T})$ is the set of $\xi$-derivatives of functions in $\mathscr{P}_{k}(\widehat{T}) .|\mathbf{U}|,\left|\underline{F_{\xi}}\right|$ and $J$ are all Lipschitz, so replacing them by their averages $|\overline{\mathbf{U}}|,\left|\bar{F}_{\xi}\right|$, and $\bar{J}$, over $\widehat{T}$, on the left-hand side introduces an error of size $h$,

$$
\begin{aligned}
& \int_{\widehat{T}}\left(\frac{|\overline{\mathbf{U}}|}{\left|\bar{F}_{\xi}\right|} \frac{\partial u}{\partial \xi}+\alpha u\right) \frac{|\overline{\mathbf{U}}|}{\left|\overline{F_{\xi}}\right|} w \bar{J} \\
& \quad \leq \int_{\widehat{T}} \frac{|\mathbf{U}|^{2}}{\left|F_{\xi}\right|^{2}} \frac{\partial v}{\partial \xi} w J+C h\left(\|\overline{\mathbf{U}} \cdot \nabla u\|_{L^{2}(T)}+\|u\|_{L^{2}(T)}\right)\|w\|_{L^{2}(\widehat{T})} .
\end{aligned}
$$

Letting $\xi_{1}(\eta)$ parameterize the left-hand side of the parent element, note that $1-e^{-C_{0} h\left(\xi-\xi_{1}(\eta)\right)} \leq C_{0} h$ for $C_{0}>0$ to be chosen later. Then

$$
\begin{aligned}
& \int_{\widehat{T}} e^{-C_{0} h\left(\xi-\xi_{1}(\eta)\right)}\left(\frac{|\overline{\mathbf{U}}|}{\left|\bar{F}_{\xi}\right|} \frac{\partial u}{\partial \xi}+\alpha u\right) \frac{|\overline{\mathbf{U}}|}{\left|\bar{F}_{\xi}\right|} w \bar{J} \\
& \quad \leq \int_{\widehat{T}} \frac{|\mathbf{U}|^{2}}{\left|F_{\xi}\right|^{2}} \frac{\partial v}{\partial \xi} w J+C h\left(\|\overline{\mathbf{U}} \cdot \nabla u\|_{L^{2}(T)}+\|u\|_{L^{2}(T)}\right)\|w\|_{L^{2}(\widehat{T})} .
\end{aligned}
$$

Bounding the first term on the right using the Cauchy-Schwarz inequality and rearranging the constant factors on the left gives

$$
\int_{\widehat{T}} e^{-C_{0} h\left(\xi-\xi_{1}(\eta)\right)}\left(\frac{\partial u}{\partial \xi}+\frac{\left|\bar{F}_{\xi}\right|}{|\overline{\mathbf{U}}|} \alpha u\right) w \leq C(h u, v)_{T}\|w\|_{L^{2}(\widehat{T})},
$$

where $C(h u, v)_{T}=C\left(\|\overline{\mathbf{U}} \cdot \nabla v\|_{L^{2}(T)}+h\left(\|\overline{\mathbf{U}} \cdot \nabla u\|_{L^{2}(T)}+\|u\|_{L^{2}(T)}\right)\right)$. Next, select $w$ as the projection of $u$ onto $\mathscr{P}_{k, \xi}(\widehat{T})$,

$$
\int_{\widehat{T}} e^{-C_{0} h\left(\xi-\xi_{1}(\eta)\right)} w z=\int_{\widehat{T}} e^{-C_{0} h\left(\xi-\xi_{1}(\eta)\right)} u z \quad \forall z \in \mathscr{P}_{k, \xi}(\widehat{T}) .
$$

Observe that

$$
\int_{\widehat{T}} e^{-C_{0} h\left(\xi-\xi_{1}(\eta)\right)} w \frac{\partial u}{\partial \xi}=\int_{\widehat{T}} e^{-C_{0} h\left(\xi-\xi_{1}(\eta)\right)} u \frac{\partial u}{\partial \xi}=\frac{1}{2} \int_{\widehat{T}} e^{-C_{0} h\left(\xi-\xi_{1}(\eta)\right)} \frac{\partial u^{2}}{\partial \xi},
$$


and $\|w\|_{L^{2}(\widehat{T})} \leq C\|u\|_{L^{2}(\widehat{T})}$, so that

$$
\int_{\widehat{T}} e^{-C_{0} h\left(\xi-\xi_{1}(\eta)\right)}\left(\frac{1}{2} \frac{\partial u^{2}}{\partial \xi}+\frac{\left|\bar{F}_{\xi}\right|}{|\overline{\mathbf{U}}|} \alpha u w\right) \leq \frac{1}{2 h} C(h u, v)_{T}^{2}+\frac{h}{2}\|u\|_{L^{2}(\widehat{T})}^{2} .
$$

Since $\left|\bar{F}_{\xi}\right| \leq C h$, it follows that

$$
\int_{\widehat{T}} e^{-C_{0} h\left(\xi-\xi_{1}(\eta)\right)}\left(\frac{\partial u^{2}}{\partial \xi}-C_{0} h u^{2}\right) \leq \frac{1}{h} C(h u, v)_{T}^{2},
$$

where $C_{0}$ is of the form $C\left(1+\|\alpha\|_{L^{\infty}(\Omega)}\right)$. The left-hand side is now an exact derivative, which may be integrated to give

$$
e^{-C_{0} h}\|u\|_{L^{2}\left(C_{2}\right)}^{2}-\|u\|_{L^{2}\left(C_{1}\right)}^{2} \leq \frac{1}{2 h} C(h u, v)_{T}^{2},
$$

where $C_{1}$ and $C_{2}$ are the left- and right-hand sides of the parent element. With $G$ denoting a stream tube, this difference relation shows that

$$
\|u\|_{L^{2}\left(C_{i}\right)}^{2} \leq \frac{C}{h} e^{C_{0} h N_{G}} \sum_{T \subset G} C(h u, v)_{T}^{2}
$$

where $C_{i}$ is any of the interelement edges within $G, N_{G}$ is the number of elements in $G$, and we have used the boundary condition $\left.u\right|_{\Gamma_{1}}=0$. Recalling the definition of $C(h u, v)_{T}$, and noting that $N_{G} \leq C / h$, gives

$$
\|u\|_{L^{2}\left(C_{i}\right)}^{2} \leq \frac{C}{h}\left(\|\overline{\mathbf{U}} \cdot \nabla v\|_{L^{2}(G)}^{2}+h^{2}\|\overline{\mathbf{U}} \cdot \nabla u\|_{L^{2}(G)}^{2}\right)
$$

where the Poincaré inequality $\|u\|_{L^{2}(G)} \leq C\|\overline{\mathbf{U}} \cdot \nabla u\|_{L^{2}(G)}$ was used. Finally, the inequality (5) shows that

$$
\|u\|_{L^{2}(T)}^{2} \leq C h^{2}\left(\|u\|_{L^{2}\left(C_{1}\right)}^{2}+\|\overline{\mathbf{U}} \cdot u\|_{L^{2}(T)}^{2}\right) .
$$

Summing over all the elements in $G$ and using the above inequality to bound the boundary terms yields

$$
\|u\|_{L^{2}(G)}^{2} \leq C\left(\|\overline{\mathbf{U}} \cdot v\|_{L^{2}(G)}^{2}+h^{2}\|\overline{\mathbf{U}} \cdot u\|_{L^{2}(G)}^{2}\right),
$$

which establishes (7).

To establish the second coercivity condition, fix $v \in U_{h}$, and suppose $a_{h}(u, v)$ $=0$ for all $u \in U_{h}$; we show that if $h$ is sufficiently small, $\overline{\mathbf{U}} \cdot \nabla v$ vanishes, so that $v=0$. If $v$ is not zero, select a streamtube where $\overline{\mathbf{U}} \cdot \nabla v$ does not vanish, and let $T$ be the last triangle for which $\overline{\mathrm{U}} \cdot \nabla v \neq 0$. Then select $u$ to be zero on all the triangles preceding $T, \overline{\mathbf{U}} \cdot \nabla u=\overline{\mathbf{U}} \cdot \nabla v$ on $T$, an arbitrary extension after $T$, and zero on all other streamtubes. It follows that

$$
0=a(u, v)=\int_{T}|\overline{\mathbf{U}} \cdot \nabla u|^{2}+\alpha u(\overline{\mathbf{U}} \cdot \nabla u)
$$




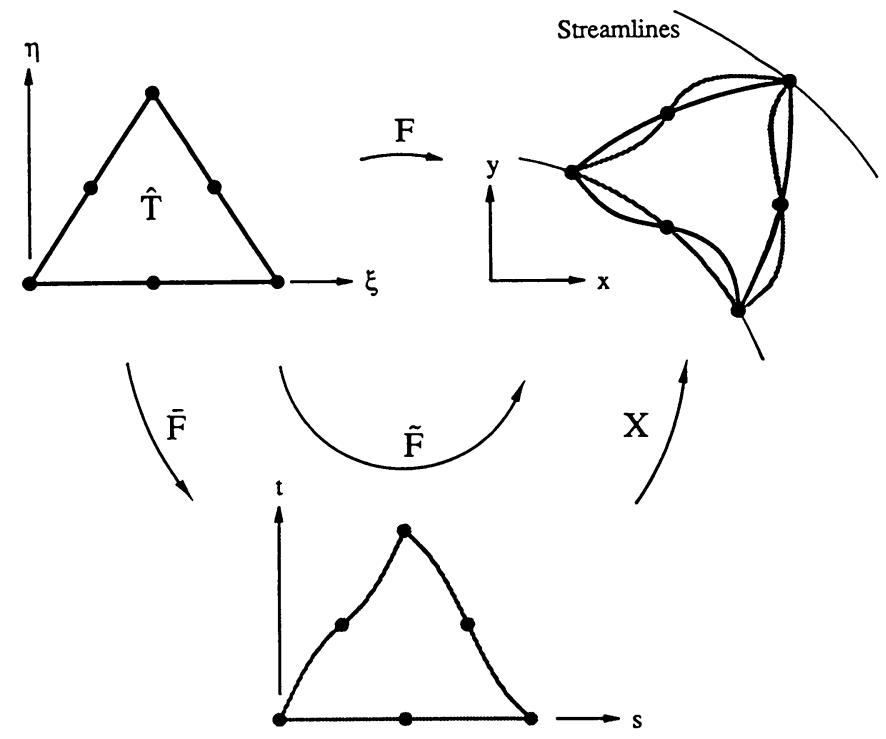

FIGURE 4

Construction of a conforming element

Since $u$ vanishes on the inflow boundary of $T$, Corollary 4 implies $\|u\|_{L^{2}(T)} \leq$ $C h\|\overline{\mathbf{U}} \cdot \nabla u\|_{L^{2}(T)}$, so that

$$
\int_{T}|\overline{\mathbf{U}} \cdot \nabla u|^{2}=\int_{T} \alpha u(\overline{\mathbf{U}} \cdot \nabla u) \leq C\|\alpha\|_{L^{\infty}(\Omega)} h \int_{T}|\overline{\mathbf{U}} \cdot \nabla u|^{2} .
$$

Clearly, $\|\overline{\mathbf{U}} \cdot \nabla u\|_{L^{2}(T)}=\|\overline{\mathbf{U}} \cdot \nabla v\|_{L^{2}(T)}=0$ if $h<1 / C\|\alpha\|_{L^{\infty}(\Omega)}$.

In order to prove that the solutions of the approximate weak problem converge to the solution of the exact weak problem, it is convenient to introduce a second mapping from the parent element to $\Omega$, chosen so that the side on the $\xi$-axis maps to a streamline of $\mathbf{U}$. This map is chosen to be of the form $\widetilde{F}=\mathbf{X} \circ \bar{F}$, where $\bar{F}$ is the isoparametric mapping that interpolates the values of the streamwise coordinates $(s, t)$ of the finite element nodes. The different maps are shown in Figure 4.

Lemma 6. Let $\left\{\mathscr{T}_{h}\right\}_{h>0}$ be a regular family of meshes, and suppose $\mathbf{U} \in C^{2}(\bar{\Omega})$, $|\mathbf{U}| \geq c_{0}>0$. Then there are constants $c$ and $C$, independent of $h$, such that

1. $c h^{2} \leq J_{\widetilde{F}} \leq C h^{2} \quad\left(J_{\widetilde{F}}\right.$ is the Jacobian of $\left.D \widetilde{F}\right)$,

2. $c h \leq\left|\widetilde{F}_{\xi}\right|,\left|\widetilde{F}_{\eta}\right|$, and $|D \widetilde{F}| \leq C h$,

3. $\left|D^{2} \widetilde{F}\right| \leq C h^{2}$.

Proof. The hypothesis on $\mathbf{U}$ guarantees that $\mathbf{X}$ is a $C^{2}$ diffeomorphism, so it follows that the $(s, t)$ meshes generated from $\left\{\mathscr{F}_{h}\right\}$ under $\mathbf{X}^{-1}$ will also be regular. Lemma 1 is then applicable to the maps $\bar{F}$. Since $\widetilde{F}=\mathbf{X} \circ \bar{F}$, the lemma follows.

Since $F$ is a polynomial map agreeing with $\widetilde{F}$ at the finite element nodes, the Bramble-Hilbert lemma [1] can be applied to give: 
Lemma 7. Let $F, \widetilde{F}: \widehat{T} \rightarrow \mathbb{R}^{2}$ be the maps from the parent element defined above and $\mathbf{U} \in C^{k+1}(\bar{\Omega})$. Then

$$
\|\widetilde{F}-F\|_{1, \infty, \widehat{T}} \leq C(\widehat{T})|\widetilde{F}|_{k+1, \infty, \widehat{T}},
$$

where $|\cdot|_{m, p, G}$ indicates the seminorm for the Sobolev space $W^{m, p}(G)$, and $k$ is the degree of the finite element interpolation polynomials.

Corollary 8. Let $\left\{\mathscr{T}_{h}\right\}$ be a regular family, and suppose $\mathbf{U} \in C^{k+1}(\bar{\Omega})$; then there is a constant, $C$, independent of $h$ such that

$$
\|\widetilde{F}-F\|_{1, \infty, \widehat{T}} \leq C h^{k+1} \text {. }
$$

When $k=1$, the corollary follows immediately from the previous two lemmas. Expansion of $D^{3} \widetilde{F}=D^{3}(\mathbf{X} \circ \bar{F})$ and using the fact that $D^{3} \bar{F}=0$ proves the result for $k=2$.

The space $U_{h}$ was chosen in order to get $\overline{\mathbf{U}} \cdot \nabla u_{h}=\left(|\mathbf{U}| /\left|F_{\xi}\right|\right) \partial \hat{u} / \partial \xi$, where $u_{h}=\hat{u} \circ F^{-1}$. The map $\widetilde{F}$ was chosen so that $\mathbf{U} \cdot \nabla \tilde{u}=\left(|\mathbf{U}| /\left|\widetilde{F}_{\xi}\right|\right) \partial \hat{u} / \partial \xi$, where $\tilde{u}=\hat{u} \circ \widetilde{F}^{-1}$. Given a function $\hat{u}: \widehat{T} \rightarrow \mathbb{R}$, we can generate $u=\hat{u} \circ F \in U_{h}$, and $\tilde{u}=\hat{u} \circ \widetilde{F} \in U$; moreover, $c\|\tilde{u}\|_{U} \leq\|u\|_{U_{h}} \leq C\|u\|_{U}$. Thus, given an approximate solution $u_{h} \in U_{h}$, we can define $\rho\left(u_{h}\right)=\tilde{u}_{h} \in U$ by $\tilde{u}_{h} \circ \widetilde{F}^{-1}=$ $u_{h} \circ F^{-1}$. This mechanism provides a means of comparing the approximate solutions $u_{h}$ with the exact solution $u$, as shown by the following Strang-type lemma.

Lemma 9. Given two Hilbert spaces $U$ and $U_{h}$, and a linear continuous map $\rho$ : $U_{h} \rightarrow U$, let $a$ and $a_{h}$ be continuous bilinear forms on $U$ and $U_{h}$, respectively, with a coercive on $\rho\left(U_{h}\right)$, with coercivity constant independent of $h$. If $f \in U^{\prime}$, $f_{h} \in U_{h}^{\prime}$, and $u, u_{h}$ satisfy $a(u, \cdot)=f(\cdot)$ and $a_{h}\left(u_{h}, \cdot\right)=f_{h}(\cdot)$, respectively, then there is a constant $C$ such that

$$
\begin{aligned}
\left\|u-u_{h}\right\|_{U} \leq C\left[\inf _{v_{h} \in U_{h}}\left\|u-v_{h}\right\|_{U}+\sup _{w_{h} \in U_{h}} \mid\right. & \frac{a\left(u_{h}, w_{h}\right)-a_{h}\left(u_{h}, w_{h}\right)}{\left\|w_{h}\right\|_{U}} \mid \\
& \left.+\sup _{w_{h} \in U_{h}}\left|\frac{f\left(w_{h}\right)-f_{h}\left(w_{h}\right)}{\left\|w_{h}\right\|_{U}}\right|\right],
\end{aligned}
$$

where we have used the identifications $\left\|w_{h}\right\|_{U} \equiv\left\|\rho w_{h}\right\|_{U}, f\left(w_{h}\right) \equiv f\left(\rho w_{h}\right)$, and $a\left(u_{h}, w_{h}\right) \equiv a\left(\rho u_{h}, \rho w_{h}\right)$.

Proof. Let $v_{h} \in U_{h}$; then

$$
a\left(u_{h}-v_{h}, w_{h}\right)=a\left(u-v_{h}, w_{h}\right)+a\left(u_{h}, w_{h}\right)-a_{h}\left(u_{h}, w_{h}\right)-f\left(w_{h}\right)+f_{h}\left(w_{h}\right) .
$$

It follows that

$$
\begin{aligned}
c\left\|u_{h}-v_{h}\right\|_{U} \leq & C\left\|u-v_{h}\right\|_{U}+\sup _{w_{h} \in U_{h}}\left|\frac{a\left(u_{h}, w_{h}\right)-a_{h}\left(u_{h}, w_{h}\right)}{\left\|w_{h}\right\|_{U}}\right| \\
& +\sup _{w_{h} \in U_{h}}\left|\frac{f\left(w_{h}\right)-f_{h}\left(w_{h}\right)}{\left\|w_{h}\right\|_{U}}\right| .
\end{aligned}
$$

An application of the triangle inequality, $\left\|u-u_{h}\right\|_{U} \leq\left\|u-v_{h}\right\|_{U}+\left\|u_{h}-v_{h}\right\|_{U}$, completes the proof. 
Remark. The proof of Theorem 5, showing that approximate weak problem (4) has a coercivity constant $c>0$ independent of $h$, can be repeated verbatim to show that the restriction of weak problem (3) to $\rho\left(U_{h}\right)$ also has a coercivity constant $c>0$ independent of $h$.

The following lemma is a modification of the Bramble-Hilbert lemma useful for the model problem where the solution may have more derivatives in one direction than another.

Lemma 10. Let $\mathscr{P}_{k}(\xi) \otimes \mathscr{P}_{k-1}(\eta)$ denote the set of polynomials formed from linear combinations of monomials of the form $\xi^{m} \eta^{n}$ with $0 \leq m \leq k$ and $0 \leq n \leq k-1$, and let $V$ denote the seminorm $|u|_{V}=\|\partial u / \partial \xi\|_{H^{k}(\widehat{T})}$. If $\Pi: V \rightarrow V$ is continuous and vanishes on $\mathscr{P}_{k}(\xi) \otimes \mathscr{P}_{k-1}(\eta)$, then there is a constant $C>0$ such that

$$
|\Pi v|_{V} \leq C\left|\frac{\partial v}{\partial \xi}\right|_{H^{k}(\widehat{T})}
$$

Corollary 11. Suppose that $u$ and $\mathbf{U} \cdot \nabla u$ are in $H^{k}(\Omega), \quad \mathbf{U} \in C^{k}(\Omega)$, and $\rho: U_{h} \rightarrow U$ is the map defined by $\rho u_{h} \circ \widetilde{F}^{-1}=u_{h} \circ F^{-1}$. Then

$$
\inf _{v_{h} \in U_{h}}\left\|u-\rho v_{h}\right\|_{U} \leq C h^{k} .
$$

Proof. When $k \geq 2, u$ is continuous, so we can define (on each element) a projection of $u$ onto $\mathscr{P}_{k}(\widehat{T})$ by $\Pi u=u$ at each of the nodes. When $k=1$, we define the $\Pi u \in \mathscr{P}_{1}(\xi) \otimes \mathscr{P}_{0}(\eta) \sim \mathscr{P}_{1}(\xi)$ as the projection onto $\mathscr{P}_{1}(\xi)$ of the average value of $u$ in the $\eta$-direction. In either instance, we get $(I-\Pi)$ vanishing on $\mathscr{P}_{k}(\xi) \otimes \mathscr{P}_{k-1}(\eta)$, so that

$$
\left\|\frac{\partial}{\partial \xi}(I-\Pi) u\right\|_{L^{2}(\widehat{T})} \leq\|(I-\Pi) u\|_{V} \leq C\left|\frac{\partial u}{\partial \xi}\right|_{H^{k}(\widehat{T})} .
$$

Upon observing that $\partial u / \partial \xi=\left(\left|\widetilde{F}_{\xi}\right| /|\mathbf{U}|\right)(\mathbf{U} \cdot \nabla u)$, the result follows from the usual scaling arguments.

Remark. For triangular elements it is not possible to construct nonzero basis functions that are constant in the $\eta$-direction and continuous in each streamtube, so the above proof for $k=1$, as stated, is valid only for isoparametric rectangular elements. However, if linear triangles are used, they can be put in pairs to form quadrilaterals, and the proof follows.

Theorem 12. Let $u \in U$ be the solution to weak problem (3) and suppose $u$ and $\mathbf{U} \cdot \nabla u \in H^{k}(\Omega)$. Assume that $\alpha$ and $f$ are Lipschitz, and have Lipschitz extensions to $\cup_{h>0} \Omega_{h} \cup \Omega$, and let $u_{h} \in U_{h}$ be the corresponding solution to the approximate weak problem (4). Define $\rho: U_{h} \rightarrow U$ by $\left(\rho u_{h}\right) \circ \widetilde{F}^{-1}=u_{h} \circ F^{-1}$, and suppose that $\left\{\mathscr{T}_{h}\right\}_{h>0}$ is a regular family, and $\mathbf{U} \in C^{k+1}(\Omega)$; then

$$
\left\|u-\rho u_{h}\right\|_{U} \leq C h^{k}
$$


Proof. We show that each of the terms on the right-hand side of (8) is suitably small. Corollary 11 shows that the projection error of $U_{h}$ into $U$ is less than $C h^{k}$, so it suffices to consider the consistency errors. This will follow from Corollary 8 , which essentially states that $F \circ \widetilde{F}^{-1}$ is close to the identity map. The estimates follow from direct calculations on the parent elements $\widehat{T}$.

We first estimate the principal term of the bilinear form:

$$
\begin{aligned}
& \int_{\Omega}\left(\overline{\mathbf{U}} \cdot \nabla u_{h}\right)\left(\overline{\mathbf{U}} \cdot \nabla w_{h}\right)-\int_{\Omega_{h}}\left(\mathbf{U} \cdot \nabla u_{h}\right)\left(\mathbf{U} \cdot \nabla w_{h}\right) \\
& =\sum_{\widehat{T}} \int_{\widehat{T}} \frac{1}{\left|F_{\xi}\right|^{2}}|\mathbf{U} \circ F|^{2} \frac{\partial \hat{u}}{\partial \xi} \frac{\partial \hat{w}}{\partial \xi} J_{F}-\frac{1}{\left|\widetilde{F}_{\xi}\right|^{2}}|\mathbf{U} \circ \widetilde{F}|^{2} \frac{\partial \hat{u}}{\partial \xi} \frac{\partial \hat{w}}{\partial \xi} J_{\widetilde{F}} \\
& =\sum_{\widehat{T}} \int_{\widehat{T}}\left\{\frac{\left|\widetilde{F}_{\xi}\right|}{\left|F_{\xi}\right|} \frac{|\mathbf{U} \circ F|}{|\mathbf{U} \circ \widetilde{F}|}\left(\frac{J_{F}}{J_{\widetilde{F}}}\right)^{\frac{1}{2}}-\frac{\left|F_{\xi}\right|}{\left|\widetilde{F}_{\xi}\right|} \frac{|\mathbf{U} \circ \widetilde{F}|}{|\mathbf{U} \circ F|}\left(\frac{J_{\widetilde{F}}}{J_{F}}\right)^{\frac{1}{2}}\right\} \\
& \quad \times \frac{1}{\left|F_{\xi}\right|}|\mathbf{U} \circ F| \frac{\partial \hat{u}}{\partial \xi} J_{F}^{\frac{1}{2}} \frac{1}{\left|\widetilde{F}_{\xi}\right|}|\mathbf{U} \circ \widetilde{F}| \frac{\partial \hat{w}}{\partial \xi} J_{\widetilde{F}}^{\frac{1}{2}} \\
& \leq C(h)\left\|u_{h}\right\|_{U_{h}}\left\|w_{h}\right\|_{U}, \quad
\end{aligned}
$$

where $C(h)$ is the supremum of the difference appearing in the curly brackets. Since $C(h)$ is the difference of a number and its reciprocal, it suffices to show that either one of them is close to unity. We show that each of the ratios involving $\mathbf{U}, F_{\xi}$ and the Jacobians differs from one by at most $C h^{k}$.

Estimates on the first two quotients follow immediately from Corollary 8:

$$
\begin{gathered}
\left|1-\frac{\left|\widetilde{F}_{\xi}\right|}{\left|F_{\xi}\right|}\right|=\frac{1}{\left|F_{\xi}\right|}\left|F_{\xi}-\widetilde{F}_{\xi}\right| \leq \frac{1}{\left|F_{\xi}\right|}|D(F-\widetilde{F})| \leq \frac{1}{c h} C h^{k+1}=C h^{k}, \\
\left|1-\frac{|\mathbf{U} \circ F|}{|\mathbf{U} \circ \widetilde{F}|}\right|=\frac{1}{|\mathbf{U} \circ \widetilde{F}|}|\mathbf{U} \circ \widetilde{F}-\mathbf{U} \circ F| \leq \frac{1}{|\mathbf{U} \circ \widetilde{F}|}\|\mathbf{U}\|_{\text {Lip }}|\widetilde{F}-F| \leq C h^{k+1} .
\end{gathered}
$$

An explicit calculation of the Jacobians on an element $\widehat{T}$ reveals

$$
\left|J_{F}-J_{\widetilde{F}}\right| \leq C|F-\widetilde{F}|_{1, \infty, \widehat{T}} \max \left(|D F|_{1, \infty, \widehat{T}},|D \widetilde{F}|_{1, \infty, \widehat{T}}\right) \leq C h^{k+2}
$$

Since each Jacobian is bounded below by $c h^{2}$, it follows that

$$
\left|1-\frac{J_{F}}{J_{\widetilde{F}}}\right| \leq C h^{k} \quad \text { and } \quad\left|1-\left(\frac{J_{F}}{J_{\widetilde{F}}}\right)^{\frac{1}{2}}\right| \leq C h^{k} \text {. }
$$

We next consider the consistency error associated with the right-hand side $f$ : 


$$
\begin{aligned}
& \int_{\Omega} f\left(\mathbf{U} \cdot \nabla w_{h}\right)-\int_{\Omega_{h}} f(\overline{\mathbf{U}} \cdot \nabla) w_{h} \\
& =\sum_{\widehat{T}} \int_{\widehat{T}}(f \circ \widetilde{F}) \frac{|\mathbf{U} \circ \widetilde{F}|}{\left|\widetilde{F}_{\xi}\right|} \frac{\partial \hat{w}}{\partial \xi} J_{\widetilde{F}}-(f \circ F) \frac{|\mathbf{U} \circ F|}{\left|F_{\xi}\right|} \frac{\partial \hat{w}}{\partial \xi} J_{F} \\
& =\sum_{\widehat{T}} \int_{\widehat{T}}\left\{[(f \circ \widetilde{F})-(f \circ F)] J_{\widetilde{F}}^{\frac{1}{2}}\right. \\
& +\left[\left(\frac{J_{\widetilde{F}}}{J_{F}}\right)^{\frac{1}{2}}-\frac{\left.\left.\left.|\mathbf{U} \circ F| \mid \frac{\left|\widetilde{F}_{\xi}\right|}{|\mathbf{U} \circ \widetilde{F}|} \frac{J_{F} \mid}{\mid F_{\xi}}\right)^{\frac{1}{2}}\right](f \circ F) J_{\widetilde{F}}^{\frac{1}{2}}\right\}}{\times \frac{|\mathbf{U} \circ \widetilde{F}|}{|\widetilde{F}|} \frac{\partial \hat{w}}{\partial \xi} J_{\widetilde{F}}^{\frac{1}{2}}}\right. \\
& \leq C h^{k}\left\|w_{h}\right\|_{U} .
\end{aligned}
$$

The last line follows since $f$ is assumed Lipschitz and $|\widetilde{F}-F| \leq C h^{k+1}$, and the second term in square brackets is small, since each of the ratios appearing is within $C h^{k}$ of being unity.

The bilinear term involving $\alpha$ is similarly small, the only difference being that the $L^{2}\left(\Omega_{h}\right)$ norm of $u_{h}$ appears, and this is bounded using Lemma 3.

Corollary 13. Let $u^{e} \in H^{1}\left(\mathbb{R}^{2}\right)$ be an extension of the exact solution $u$. Under the hypotheses of the previous theorem, $\left\|u^{e}-u_{h}\right\|_{L^{2}\left(\Omega_{h}\right)} \leq C h^{k}$.

Proof. By the triangle inequality,

$$
\left\|u^{e}-u_{h}\right\|_{L^{2}\left(\Omega_{h}\right)} \leq\left\|u^{e}-\rho^{-1}(u)\right\|_{L^{2}\left(\Omega_{h}\right)}+\left\|\rho^{-1}(u)-u_{h}\right\|_{L^{2}\left(\Omega_{h}\right)} .
$$

Recall that $\rho^{-1}(u)(x)=u \circ \widetilde{F} \circ F^{-1}(x)$; a change of variables then shows that

$$
\left\|\rho^{-1}(u)-u_{h}\right\|_{L^{2}\left(\Omega_{h}\right)}^{2}=\int_{\Omega}\left|u-\rho\left(u_{h}\right)\right|^{2} \frac{J_{\widetilde{F}}}{J_{F}} \leq\left\|u-\rho\left(u_{h}\right)^{2}\right\|_{L^{2}\left(\Omega_{h}\right.}^{2} \leq C h^{2} k .
$$

Since $\rho^{-1}\left(u^{e}\right)=\rho^{-1}(u)$ on $\Omega_{h}$, it follows that for $x \in \Omega_{h}$,

(9) $u^{e}(x)-u\left(\widetilde{F} \circ F^{-1}(x)\right)=\int_{0}^{1} \nabla u^{e}\left(x+t\left(\widetilde{F} \circ F^{-1}(x)-x\right)\right) \cdot\left(\widetilde{F} \circ F^{-1}(x)-x\right)$.

Defining $H(x)=\widetilde{F} \circ F^{-1}(x)-x$, we see from Lemmas 4, 6, and Corollary 8 that $|H(x)| \leq C h^{k+1}$ and $|D H| \leq C h^{k}$. It follows that, if $h$ is sufficiently small, $|D H|<1$, so that $x+t H(x)$ is a diffeomorphism. Squaring both sides of (9), and integrating yields

$$
\int_{\Omega_{h}}\left|u^{e}(x)-u\left(\widetilde{F} \circ F^{-1}(x)\right)\right|^{2} \leq C h^{2(k+1)} \int_{\Omega_{h}} \int_{0}^{1}\left|\nabla u^{e}(x+t H(x))\right|^{2} d t d x .
$$

Interchanging the order of integration, and introducing a change of variables $y=x+t H(x)$, shows that

$$
\left\|u^{e}-\rho^{-1}(u)\right\|_{L^{2}\left(\Omega_{h}\right)} \leq C h^{k+1}\left\|u^{e}\right\|_{H^{1}\left(\mathbf{R}^{2}\right)} .
$$


Remark. (i) If $u$ and $u_{h}$ were extended to zero on $\mathbb{R}^{2}$, then $\left\|u-u_{h}\right\|_{L^{2}\left(\mathbb{R}^{2}\right)}$ would involve terms of the form $\|u\|_{L^{2}\left(\Omega \backslash \Omega_{h}\right)}$ and $\left\|u^{e}\right\|_{L^{2}\left(\Omega_{h} \backslash \Omega\right)}$. The sets $\Omega \backslash \Omega_{h}$ and $\Omega_{h} \backslash \Omega$ will have measure bounded by $C h^{k+1}$ (we are assuming that $\Gamma_{1}$ and $\Gamma_{2}$ are triangulated exactly so that the remaining boundary, being tangent to the flow, is as smooth as $\mathbf{U})$. The Sobolev embedding theorem in $\mathbb{R}^{2}$ implies $\|u\|_{L^{2}(E)} \leq C(p)\|u\|_{H^{1}(\Omega)}|E|^{(1-1 / p) / 2}$, for $E \subset \Omega, 1 \leq p<\infty$. It follows that $\left\|u^{e}\right\|_{L^{2}\left(\Omega_{h} \backslash \Omega\right)},\|u\|_{L^{2}\left(\Omega \backslash \Omega_{h}\right)} \leq C(\varepsilon) h^{(k+1) / 2-\varepsilon}$. For linear elements this is essentially as small as $\left\|u^{e}-u_{h}\right\|_{L^{2}\left(\Omega_{h}\right)}$; however, for quadratic elements this is half a power of $h$ smaller than $\left\|u^{e}-u_{h}\right\|_{L^{2}\left(\Omega_{h}\right)}$.

(ii) The rates of convergence given in Theorem 12 are preserved for solutions which have a finite number of jump discontinuities, provided interelement nodes are positioned where the jumps meet $\Gamma_{1}$.

\section{Application to an ACOUSTICS PROBLEM}

In [11] the following weak problem was considered: find $(\phi, \psi, h, a) \in$ $\mathscr{H} \equiv H_{\Gamma_{1}}^{1}(\Omega) \times H_{0}^{1}(\Omega) \times H_{\Gamma_{1}}^{1}(\Omega) \times U$ such that

$$
\begin{aligned}
\mathbf{a}\left[\left(\phi-\phi_{0}, \psi, h-h_{0}, a-a_{0}\right),(\eta, \xi, g, b)\right]=F(\eta, \xi, g, b) \\
\forall(\eta, \xi, g, b) \in \mathscr{H} .
\end{aligned}
$$

Here, $H_{\Gamma_{1}}^{1}(\Omega)$ is the subset of $H^{1}(\Omega)$ which have zero trace on $\Gamma_{1}$, and the sesquilinear form $\mathbf{a}: \mathscr{H} \times \mathscr{H} \rightarrow \mathbb{C}$ is given by

$$
\begin{aligned}
& \mathbf{a}[(\phi, \psi, h, a),(\eta, \xi, g, b)] \\
&=\int_{\Omega}\left\{\left(R \mathbf{u}+\frac{1}{C^{2}} \mathbf{U} p\right) \cdot \nabla \bar{\eta}-\frac{i k}{C^{2}} p \bar{\eta}+\nabla \psi \cdot \nabla \bar{\xi}-\omega \bar{\xi}\right. \\
&\quad+\nabla h \cdot \nabla \bar{g}+(\omega \mathbf{U}+R A \mathbf{u}) \cdot \operatorname{curl} \bar{g}+(i k a+\mathbf{U} \cdot \nabla a+\mathbf{u} \cdot \nabla A) \mathbf{U} \cdot \nabla \bar{b}\},
\end{aligned}
$$

where the overbar indicates complex conjugate, and for a scalar function $g$, curl $g=(\partial g / \partial y,-\partial g / \partial x)$. The linear functional $F: \mathscr{H} \rightarrow \mathbb{C}$ is defined by

$$
F(\eta, \xi, g, b)=\int_{\Gamma_{2}}\left(l_{1} \bar{\eta}+l_{2} \bar{g}\right) .
$$

In the above, $k$ is a constant, and $R, \mathbf{U}, C$, and $A$ are all specified functions on $\Omega$, and $l_{1}$ and $l_{2}$ are specified functions on $\Gamma_{2}$. The functions $\phi_{0}, h_{0}$, and $a_{0}$ define boundary values on $\Gamma_{1}$, and are assumed to vanish on $\Gamma_{2}$. The auxiliary quantities $\mathbf{u}, \omega$, and $p$ are calculated from $(\phi, \psi, h, a)$ according to

$$
\mathbf{u}=\nabla \phi+\operatorname{curl} \psi, \quad p=R(h-i k \phi-\mathbf{U} \cdot \mathbf{u}), \quad \omega=R a+\frac{A}{C^{2}} p .
$$

It was shown in [11] that this problem is well posed under suitable assumptions on the data provided the frequency $k$ and vorticity $A$ were sufficiently small. In this situation, the coercivity constants depend upon Poincare constants only. This implies that the discrete problems will be well posed provided the approximating subspaces satisfy Poincaré inequalities with constants bounded independently of $h$.

Using the ideas from the previous section, we can show that approximate solutions calculated on meshes aligned with the streamlines of $\mathbf{U}$ will converge 
at optimal rates to the exact solution. We first construct a finite element space of functions $\mathscr{H}_{h}$ using the mesh $\mathscr{T}_{h}$ with standard finite element basis functions for the first three components, and $U_{h}$ for the last component. As with the model problem, there is a map $\rho: \mathscr{H}_{h} \rightarrow \mathscr{H}$ formed by composition with $F \circ \widetilde{F}^{-1}$. Weak problem (1) is then approximated in the natural fashion, except that we replace $\mathbf{U}$ by $\overline{\mathbf{U}}$.

Theorem 14. Let $\phi, \psi, h \in H^{k+1}(\Omega)$ and $a, \mathbf{U} \cdot \nabla a \in H^{k}(\Omega)$ be the solution of the acoustics problem (10) given in [11]. Assume that all the coefficients $R$, $C, \nabla A$, etc. have Lipschitz extensions to $\bigcup_{h>0} \Omega_{h} \cup \Omega$, and $\left\{\mathscr{T}_{h}\right\}_{h>0}$ is a regular family of meshes aligned with the streamlines of $\mathbf{U} \in C^{k+1}(\Omega)$ as described in the previous section. If $(\phi, \psi, h, a)_{h} \in \mathscr{H}_{h}$ denotes the solution of the approximate weak problem, then

$$
\left\|\rho(\phi, \psi, h, a)_{h}-(\phi, \psi, h, a)\right\|_{\mathscr{H}} \leq C h^{k} .
$$

Proof. The proof of this theorem uses Lemma 9 in the same way as was done for Theorem 12. The first term on the right of equation (8) is of order $C h^{k}$ by virtue of standard interpolation results and Corollary 11 , and the second term involving a and $\mathbf{a}_{h}$ will be small, since $F \circ \widetilde{F}^{-1}$ is almost the identity. In order to show this, recall that since $\mathbf{a}$ is bilinear, we only have to consider products of pairs of functions, products of pairs of derivatives, or the product of a function and a derivative, all possibly multiplied by a Lipschitz coefficient. We give a typical calculation for a term of the latter type. One can generically write such a term as $u_{h}\left(\boldsymbol{\alpha} \cdot \nabla v_{h}\right)$ where $\boldsymbol{\alpha}$ is the Lipschitz coefficient. We use the notation $\widehat{\nabla}$ to denote the gradient on the parent element $\widehat{T}$ and use the change of variables formula

$$
\widehat{\nabla}=[D F] \nabla, \quad \nabla=\frac{1}{J_{F}}[\operatorname{adj} F] \widehat{\nabla},
$$

to calculate terms in $\mathbf{a}_{h}$, and for terms in a we use $\widetilde{F}$ in place of $F$ :

$$
\begin{aligned}
& \int_{\Omega}\left(\rho u_{h}\right) \boldsymbol{\alpha} \cdot \nabla\left(\rho v_{h}\right)-\int_{\Omega_{h}} u_{h}\left(\boldsymbol{\alpha} \cdot \nabla v_{h}\right) \\
& =\sum_{T} \int_{\widehat{T}} \hat{u}\{(\boldsymbol{\alpha} \circ \widetilde{F})[\operatorname{adj} \widetilde{F}]-(\boldsymbol{\alpha} \circ F)[\operatorname{adj} F]\} \widehat{\nabla} \hat{v} \\
& =\sum_{T} \int_{\widehat{T}} \hat{u}\left\{J_{F}^{\frac{1}{2}}(\boldsymbol{\alpha} \circ \widetilde{F}-\boldsymbol{\alpha} \circ F)\left(\frac{J_{\widetilde{F}}}{J_{F}}\right)^{\frac{1}{2}}[\operatorname{adj} \widetilde{F}] \frac{1}{J_{\widetilde{F}}^{\frac{1}{2}}}\right. \\
& \left.\quad+J_{F}^{\frac{1}{2}}(\boldsymbol{\alpha} \circ F)([\operatorname{adj} \widetilde{F}]-[\operatorname{adj} F]) \frac{1}{J_{F}^{\frac{1}{2}}}\right\} \hat{\nabla} \hat{v} \\
& \leq \sum_{T} C\left\|u_{h}\right\|_{L^{2}(T)}\left\{h^{k+1}\|\nabla v\|_{L^{2}(\tilde{T})}+h^{k}\|\widehat{\nabla} \hat{v}\|_{L^{2}(\widehat{T})}\right\} \\
& \leq \sum_{T} C h^{k}\left\|u_{h}\right\|_{L^{2}(T)}\left\|\nabla v_{h}\right\|_{L^{2}(\tilde{T})},
\end{aligned}
$$

where we have used the notation $T=F(\widehat{T})$ and $\widetilde{T}=\widetilde{F}(\widehat{T})$. The last line follows from the inequality $\|\widehat{\nabla} \hat{v}\|_{L^{2}(\widehat{T})} \leq C\left\|\nabla v_{h}\right\|_{L^{2}(\widetilde{T})}$. 


\section{APPENDIX}

\section{A. Streamwise coordinates}

In this Appendix, we discuss the technical details that justify the assumption that there is a well-defined streamwise coordinate system $(s, t)$ when $\Omega$ is a Lipschitz simply connected domain and $\mathbf{U} \in C^{1}(\bar{\Omega})$ is a nonvanishing velocity field.

Given a point $\mathbf{x}(t) \in \Omega$, we can solve the differential equation

$$
\frac{\partial \mathbf{X}}{\partial s}(s, t)=\mathbf{U}(\mathbf{X}(s, t)), \quad \mathbf{X}(0, t)=\mathbf{x}(t) .
$$

The assumptions on $\mathbf{U}$ and $\Omega$ allow a $C^{1}$ extension of $\mathbf{U}$ to a neighborhood of $\bar{\Omega}$. Since $\mathbf{U}$ is Lipschitz on $\bar{\Omega}$, maximal solutions of (1) exist in $\bar{\Omega}$, i.e., integral curves of (1) exist and remain in $\bar{\Omega}$ for $s$ in some maximal interval. If the maximal interval were of infinite length, the Poincaré-Bendixson Theorem [5, p. 151] would imply the existence of a periodic orbit in $\bar{\Omega}$ bounded by a Jordan curve. However, a nonvanishing vector field defined on the interior of, and tangent to, a Jordan curve must have a zero [5, pp. 147-149], contradicting the assumption $\mathbf{U}$ is never zero in $\bar{\Omega}$. This argument shows that each maximal trajectory of (A.1) must begin and end on $\partial \Omega$.

Definition 15. The inflow boundary $\Gamma_{1}$ corresponding to $U$ is the union of the points on $\partial \Omega$ where the backward integral curves of (A.1) emanate from $\bar{\Omega}$. Similarly, the outflow boundary is defined to be the point where the forward integral curves emanate from $\bar{\Omega}$.

The reason for considering maximal solutions in $\bar{\Omega}$ as opposed to $\Omega$ is to allow for the possibility that a streamline may be tangential to the boundary at a point. This also allows portions of the boundary to consist of streamlines which may also enter the interior.

The uniqueness theorem for ordinary differential equations shows that every point on $\Omega$ is on a unique streamline of the flow $\mathbf{U}$, and these curves may be indexed by the points on $\Gamma_{1}$. We wish to construct a coordinate system aligned with the streamlines. It is easy to construct examples where $\Gamma_{1}$ has an infinite number of components (e.g., consider a boundary curve given by $y=x^{2} \sin (1 / x)$ and $\mathbf{U}$ parallel to $\left.x\right)$. This would be a rare circumstance for a physical flow, and would result in numerous additional problems from a numerical standpoint, so it is assumed that $\Gamma_{1}$ has a finite number of nondegenerate components. When $\Gamma_{1}$ has a finite number of components, equation (1) can be considered separately on each component, so we need only consider the construction of a coordinate system when one component is involved.

When $\Gamma_{1}$ has only one component, it is homeomorphic to an interval, so can be parameterized by arc length $t$. Letting $t=0$ at the first point of the interval, we construct the mapping $(x, y)=\mathbf{X}(s, t)$ by letting $(x, y)$ be the point in $\Omega$ at position $s$ on the integral curve of (1) emanating from the point corresponding to $t$ on $\Gamma_{1}$. By construction, $\mathbf{X}$ will be injective, and since the solution of (A.1) depends continuously upon the initial data, $\mathbf{X}$ is continuous. The theorem on the invariance of domains guarantees that this map is then a homeomorphism. 


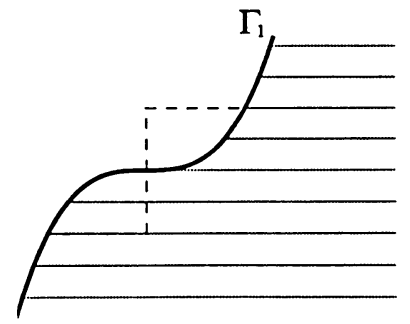

FIGURE 5

Parameterizing the streamlines

If $\Gamma_{1}$ is smooth, then $\mathbf{X}$ is smooth, so $\mathbf{X}$ will be a diffeomorphism provided the Jacobian never vanishes. With $J$ denoting the Jacobian of $\mathbf{X}$, the construction of the inflow boundary guarantees that $J \geq 0$. A classical result from continuum mechanics [9] states that along the integral curves of (A.1), $J$ satisfies

$$
\frac{\partial J}{\partial s}=(\nabla \cdot \mathbf{U}) J
$$

so that $J(s, t) \geq \exp (-C s) J(0, t)$, where $\nabla \cdot \mathbf{U} \geq-C$. It follows that if $J$ is bounded away from zero on $\Gamma_{1}$, then $J$ is bounded away from zero throughout $\Omega$. The inverse function theorem then guarantees that $\mathbf{X}$ will be a $C^{k}$ diffeomorphism whenever $\mathbf{U} \in C^{k}(\Omega)$ and $\Gamma_{1}$ is $C^{k}$. If $\mathbf{U}$ is tangential to $\Gamma_{1}$ at an isolated point, then $J(0, t)=0$. In this situation, the construction of the transverse variable $t$ can be modified as indicated in Figure 5. The idea is to extend $\mathbf{U}$ to a neighborhood of the offending point, construct an arc perpendicular to $\mathbf{U}$ through this point, and let this arc parameterize the streamlines. An appropriate parameterization of the arc will result in global $C^{k}$ dependence of $\mathbf{X}$ upon $t$, and by construction, $J$ is bounded away from zero near the point in question.

If $\Gamma_{1}$ is only Lipschitz, and the angle between $U$ and the tangent is bounded away from zero, a.e., $\Gamma_{1}$ can be approximated by a smooth curve as follows. Extend $\mathbf{U}$ to a neighborhood of $\bar{\Omega}$, and extend $\Gamma_{1}$ at each end with extensions nontangential to $\mathbf{U}$. If $\mathbf{x}(\cdot)$ is the arc length parameterization of $\Gamma_{1}$, a calculation shows that mollifying $\mathbf{x}(\cdot)$ will yield a $C^{\infty}$ approximation $\mathbf{x}_{\varepsilon}(\cdot)$ such that the angle between the tangent and $\mathbf{U}$ is bounded away from zero (provided $\varepsilon$ is suitably small). This approximating curve can then be used to specify the initial condition for $\mathbf{X}$ on each streamline. Note that with the above modifications, the boundary $\Gamma_{1}$ may no longer correspond to $s=0$, but this is of no consequence.

A.1. Solution of the hyperbolic equation. In this section, we wish to show that (1) has a solution in the space $U$ introduced in $\S 2$, when the right-hand side $f \in$ $L^{2}(\Omega)$. We assume that the inflow boundary $\Gamma_{1}$ has only one component, and that the map $(x, y)=\mathbf{X}(s, t)$ is a $C^{1}$ diffeomorphism, and that the Jacobian is bounded away from zero. By transforming to the $(s, t)$ variables, it is clear that the Poincare inequality, $\|u\|_{L^{2}(\Omega)} \leq C\|(\mathbf{U} \cdot \nabla) u\|_{L^{2}(\Omega)}$, holds for elements $u \in U$. 
Lemma 16. Let $\mathbf{U} \in C^{1}(\bar{\Omega})$ be a nonvanishing vector field, and suppose that the mapping to the streamwise coordinates is a $C^{1}$ diffeomorphism with Jacobian bounded away from zero. Let $\alpha \in L^{\infty}(\Omega)$; then equation $(\mathbf{U} \cdot \nabla) u+\alpha u=f$ has a solution $u \in U$ satisfying $\|u\|_{U} \leq C\|f\|_{L^{2}(\Omega)}$.

Proof. Since $C^{1}(\bar{\Omega})$ is dense in $L^{2}(\Omega)$ it suffices to consider $f \in C^{1}(\bar{\Omega})$. Transforming to the streamwise coordinates leads to the equation

$$
\frac{\partial u}{\partial s}+\alpha u=f
$$

subject to $u=0$ on $\Gamma_{1}$. Clearly, this is a linear ordinary differential equation for each fixed value of $t$, which can be solved by Picard's theorem. Multiplying this equation by $u$ gives

$$
\frac{1}{2} \frac{\partial}{\partial s}|u|^{2}+\alpha|u|^{2}=f u
$$

so that

$$
|u(s, t)|^{2} \leq C\left(\|\alpha\|_{L^{\infty}(\Omega)}, \operatorname{diam}(\widehat{\Omega})\right) \int_{0}^{s}|f|(\xi, t) d \xi
$$

$\left(\widehat{\Omega}=\mathbf{X}^{-1}(\Omega)\right)$. It follows that $\|u\|_{L^{2}(\widehat{\Omega})} \leq C\|f\|_{L^{2}(\widehat{\Omega})}$. The equation itself then implies that

$$
\left\|\frac{\partial u}{\partial s}\right\|_{L^{2}(\widehat{\Omega})} \leq\|f\|_{L^{2}(\widehat{\Omega})}+\|\alpha\|_{L^{\infty}(\Omega)}\|u\|_{L^{2}(\widehat{\Omega})} \leq C\|f\|_{L^{2}(\widehat{\Omega})} .
$$

Transforming these estimates back to $\Omega$ completes the proof.

\section{BIBLIOGRAPHY}

1. J. H. Bramble and S. R. Hilbert, Estimation of linear functionals on Sobolev spaces with application to Fourier transforms and spline interpolation, SIAM J. Numer. Anal. 7 (1979), 112-124.

2. P. G. Ciarlet, The finite element method for elliptic problems, North-Holland, Amsterdam, 1978.

3. J. Douglas, Jr. and T. F. Russel, Numerical methods for convection-dominated diffusion problems based on combining the method of characteristics with finite element or finite difference procedures, SIAM J. Numer. Anal. 19 (1982), 871-885.

4. R. S. Falk and G. R. Richter, Analysis of a continuous finite element method for hyperbolic equations, SIAM J. Numer. Anal. 24 (1987), 257-278.

5. P. Hartman, Ordinary differential equations, Wiley, New York, 1964.

6. C. Johnson, Streamline diffusion methods for problems in fluid mechanics, Finite Elements in Fluids, Vol. 6 (R. H. Gallagher, G. F. Carey, J. T. Oden, and O. C Zienkiewicz, eds.), Wiley, Chichester, 1985.

7. C. Johnson and J. Pitkäranta, An analysis of the discontinuous Galerkin method for a scalar hyperbolic equation, Math. Comp. 46 (1986), 1-26.

8. P. Lasaint and P. A. Raviart, On a finite element method for solving the neutron transport equation, Mathematical Aspects of Finite Elements in Partial Differential Equations (C. de Boor, ed.), Academic Press, 1974, pp. 89-123.

9. J. Serrin, Mathematical principles of classical fluid mechanics, Handbuch der Physik, Volume VIII/I, Springer-Verlag, Berlin, 1959. 
10. L. B. Wahlbin, A dissipative Galerkin method for the numerical solution of first order hyperbolic equations, Mathematical Aspects of the Finite Element Method in Partial Differential Equations (C. de Boor, ed.), Academic Press, 1974, pp. 147-170.

11. N. J. Walkington, Acoustic wave propagation through flows with vorticity, SIAM J. Numer. Anal. 25 (1988), 533-539.

12. R. Winther, $A$ stable finite element method for initial-boundary value problems for first-order hyperbolic systems, Math. Comp. 36 (1981), 65-68.

Department of Mathematics, Carnegie Mellon University, Pittsburgh, Pennsylvania 15213

E-mail address:nw0y@andrew.cmu.edu 